\title{
Intérêt de la technique de précipitation au sulfate de dextrane dans la détermination du cholestérol-LDL
}

\author{
M. GOMINA ASSOUMANOU ${ }^{1}$, C. C. AGNIWO ${ }^{1}$, M. M. SOUMANOU ${ }^{2}$ et \\ S. A. AKPONA ${ }^{1 *}$ \\ ${ }^{1}$ UERS de Biochimie et de Biologie Moléculaire, Faculté de Médecine, Université de Parakou, BP : 123 \\ Parakou, République du Bénin. \\ ${ }^{2}$ Ecole Polytechnique d'Abomey-Calavi, Université d'Abomey-Calavi, BP : 526 Abomey-Calavi, République \\ du Bénin. \\ *Auteur correspondant; E-mail : akponasimon@yahoo.fr; Tel : +229 95524484
}

\section{RESUME}

Le but de ce travail était de déterminer les valeurs du cholestérol-LDL (C-LDL) dans le sérum par la technique de précipitation au sulfate de dextrane et de les corréler avec celles obtenues par la formule de Friedewald. Les sera de 207 sujets (113 hommes, 94 femmes) volontaires d'âge moyen 41,49 \pm 3,08 ans ont été analysés en double aveugle par les deux méthodes. Le cholestérol total (CT), le cholestérol-HDL (C-HDL) et les triglycérides (TG) ont été dosés par la méthode enzymatique en point final suivi du calcul du C-LDL par la formule de Friedewald. La précipitation du C-LDL a été réalisée au sulfate de dextrane et son dosage effectué directement sur la fraction précipitée par la méthode enzymatique. Les valeurs moyennes du C-LDL obtenues étaient de 2,67 $\pm 0,58 \mathrm{mmol} / \mathrm{l}$ par la formule de Friedewald et de 2,93 $\pm 0,68 \mathrm{mmol} / \mathrm{l}$ par la technique de précipitation au sulfate de dextrane. Les deux techniques avaient une corrélation positive avec un coefficient de corrélation $\mathrm{r}=+0,905$. La technique de précipitation au sulfate de dextrane peut donc être utilisée pour le dosage du C-LDL.

(c) 2010 International Formulae Group. All rights reserved.

Mots clés : Friedewald, sérum, enzymatique, corrélation.

\section{INTRODUCTION}

La détermination du taux plasmatique des lipoprotéines est importante dans le diagnostic des anomalies primitives et secondaires de transport du cholestérol ainsi que de l'évaluation du risque de survenue de maladies athéromateuses (Grundy et al., 2000). Trois lipoprotéines majeures ont été identifiées dans le plasma, les VLDL, les LDL et les HDL, différentes par leur composition en lipides et en apoprotéines. Il existe une corrélation positive entre le risque prématuré de survenue des maladies coronariennes et le taux plasmatique du cholestérol total et du cholestérol-LDL (C-LDL) (Wood et al., 1998 ; Grundy et al., 2000).

Les recommandations internationales soulignent l'importance de la détermination du C-LDL dans la prise en charge et le suivi des patients à risque cardiovasculaire. Dans la plupart des études et en pratique courante, le C-LDL est estimé par la formule de 
Friedewald et al. (1972). L'exactitude de ce calcul dépend étroitement de la précision des paramètres pris en compte [cholestérol total (CT), triglycérides (TG) et cholestérol HDL (C-HDL)] et le C-LDL calculé n'est plus corrélé aux méthodes de référence quand les TG sont supérieurs à $4,5 \mathrm{mmol} / \mathrm{l}$ ou en présence de lipoprotéines anormales (McNamara et al., 1990; Demacker et al., 1996; Nauck et al., 2002; Renard et al., 2002). C'est également pour pallier les inexactitudes de la formule de Friedewald que d'autres équations ont été proposées, parmi elles, la formule de Planella et al. (1997), mettant en jeu l'apolipoprotéine B dont la standardisation est plus accomplie que celle du C-HDL (Marcovina et al., 1994). Ces équations présentent le même inconvénient à savoir l'obligation de déterminer simultanément plusieurs paramètres et d'introduire autant de facteurs d'erreur potentiels. Ces restrictions et incertitudes du C-LDL calculé ont conduit au développement récent des méthodes directes, homogènes et adaptables sur les analyseurs de biochimie usuels (Bayer et al., 2005; Iwasaki et al., 2006). Dans les pays du tiers monde, les méthodes directes de détermination de C-LDL sont peu connues en raison de la non disponibilité des analyseurs dans les laboratoires de chimie clinique.

L'objectif de ce travail était de déterminer les valeurs du C-LDL dans le sérum par la technique de précipitation au sulfate de dextrane et de les corréler avec celles obtenues par la formule de Friedewald.

\section{MATERIEL ET METHODES Population d'étude}

Notre population d'étude était constituée de 207 sujets (113 hommes contre 94 femmes) venus au laboratoire de biochimie du Centre Hospitalier Départemental du Borgou (République du Bénin) pour un bilan biologique. Après un consentement éclairé, des mesures anthropométriques ont été effectuées, suivies d'une prise de sang veineux $(4 \mathrm{ml})$ dans un tube sans anticoagulant chez des sujets à jeun depuis 12 heures de temps. Les échantillons de sang prélevés ont été centrifugés à $3000 \mathrm{rpm}$ pendant 10 minutes à $30{ }^{\circ} \mathrm{C}$ et les dosages réalisés le même jour sur les sérums. Les caractéristiques biométriques de la population d'étude figurent au Tableau 1.

\section{Réactifs}

Les réactifs tels que le sulfate de dextrane à haut poids moléculaire, le chlorure de calcium anhydre $\left(\mathrm{CaCl}_{2}\right)$ et le chlorure de sodium $(\mathrm{NaCl})$ ont été procurés du laboratoire Fisher Scientific Labosi. Les kits prêts à l'emploi pour le dosage du cholestérol total, du C-HDL et des TG ont été acquis du laboratoire Erba Mannheim (www.erbamannheim.com).

\section{Préparation des solutions de travail}

La solution de sulfate de dextrane à $5 \%$ a été préparée en dissolvant 1 gramme de sulfate de dextrane à haut poids moléculaire dans 20 millilitres d'eau distillée. La solution de chlorure de calcium $\left(\mathrm{CaCl}_{2}\right)$ à $11,10 \%$ a été obtenue en dissolvant 22,2 grammes de $\mathrm{CaCl}_{2}$ anhydre dans 200 millilitres d'eau distillée. La solution de chlorure de sodium $(\mathrm{NaCl}) 4 \mathrm{M}$ a été préparée en dissolvant 23,37 grammes de $\mathrm{NaCl}$ dans 100 millilitres d'eau distillée. Ces solutions étaient préparées chaque jour pour utilisation.

\section{Réalisation des dosages}

Le cholestérol total dans le sérum a été dosé par la technique enzymatique en point final dont le principe est le suivant: après hydrolyse du cholestérol estérifié par une estérase, le cholestérol libéré est oxydé par une oxydase en $\mathrm{H}_{2} \mathrm{O}_{2}$ et une cholest-4-ène-3one qui transforment un chromogène incolore en chromogène coloré. L'intensité de la coloration obtenue est proportionnelle à la quantité de cholestérol dans la prise d'essai. 
Selon les recommandations du fabricant, 1000 $\mu \mathrm{l}$ de réactif (Erba Mannheim, www.erbamannheim.com) et $10 \mu \mathrm{l}$ de sérum ont été mélangés dans un tube à hémolyse et le tout incubé au bain marie à $37{ }^{\circ} \mathrm{C}$ pendant 10 minutes, puis la lecture a été faite au spectrophotomètre (Kayto Chemistry Analyser RT-1904 C) à 500 nm contre un blanc réactif contenant $10 \mu \mathrm{l}$ d'eau distillée après étalonnage par le standard.

Le C-HDL a été dosé, après séparation par précipitation, par la méthode enzymatique. Le principe du dosage est le suivant: les chylomicrons, le C-LDL et le cholestérolVLDL du sérum sont précipités par le phosphotungstate en présence de cations divalents comme le $\mathrm{Mg}^{++}$. Le C-HDL dans le surnageant est dosé par la méthode enzymatique en point final. La séparation du C-HDL des autres lipoprotéines a été réalisée comme suit: dans un tube à hémolyse, nous avons mis $500 \mu \mathrm{l}$ de réactif de précipitation (Erba Mannheim, www.erbamannheim.com) et $250 \mu \mathrm{l}$ de sérum puis nous avons centrifugé à $4000 \mathrm{rpm}$ pendant 10minutes. Le C-HDL a été dosé avec $50 \mu \mathrm{l}$ de surnageant et $1000 \mu \mathrm{l}$ de réactif (Erba Mannheim, www.erbamannheim.com) que nous avons incubé dans un tube à hémolyse à $37{ }^{\circ} \mathrm{C}$ pendant 10 minutes et la coloration apparue lue au spectrophotomètre (Kayto Chemistry Analyser RT-1904 C) à 505 nm contre un blanc réactif contenant $50 \mu \mathrm{l}$ d'eau distillée et après étalonnage par le standard.

Le dosage des TG a été effectué en utilisant la méthode de McGowan et al. (1983). Brièvement, $1000 \mu \mathrm{l}$ du réactif (Erba Mannheim, www.erbamannheim.com) et 10 $\mu \mathrm{l}$ de sérum ont été mélangés dans un tube à hémolyse et incubés à $37{ }^{\circ} \mathrm{C}$ dans un bain marie pendant 10 minutes. La densité optique de la coloration obtenue a été lue au spectrophotomètre (Kayto Chemistry Analyser RT-1904 C) à 505 nm contre un blanc réactif contenant de l'eau distillée et après étalonnage par le standard.

Les valeurs obtenues à l'issue de ces dosages ont permis de calculer la valeur du CLDL dans chaque sérum par la formule de Friedewald $[\mathrm{C}-\mathrm{LDL}=(\mathrm{CT})-(\mathrm{C}-\mathrm{HDL})-$ $\mathrm{TG} / 2,2$ en $\mathrm{mmol} / \mathrm{l}]$.

Dosage du C-LDL par la technique de précipitation au sulfate de dextrane: cette technique repose sur le principe de séparation mettant à profit la précipitation spécifique des $\beta$-lipoprotéines par le sulfate de dextrane en un milieu chloruré calcique de force ionique bien déterminée. Dans un tube à hémolyse, nous avons introduit $1000 \mu \mathrm{l}$ de sérum, $40 \mu \mathrm{l}$ de la solution de sulfate de dextrane à $5 \%$ et $100 \mu \mathrm{l}$ de la solution de $\mathrm{CaCl}_{2}$ à $11,10 \%$. Après une incubation de 15 minutes à la température du laboratoire $\left(20-25{ }^{\circ} \mathrm{C}\right)$, nous avons centrifugé à $3500 \mathrm{rpm}$ pendant 15 minutes. Après décantation suivi du nettoyage délicat des parois du tube à l'eau distillée pour s'assurer de l'élimination totale du surnageant, le culot de centrifugation a été redissout dans $500 \mu \mathrm{l}$ de solution de $\mathrm{NaCl} 4$ M. Le dosage du C-LDL dans la fraction précipitée a été réalisé avec $10 \mu \mathrm{l}$ de la suspension obtenue selon le même protocole que le dosage du cholestérol total décrit plus haut.

\section{Analyse statistique \\ L'analyse des résultats a été effectuée à l'aide du logiciel de statistiques Epi-Info version 6 (CDC, Atlanta). La corrélation entre la méthode de détermination de C-LDL par la formule de Friedewald et la technique de précipitation au sulfate de dextrane a été étudiée par le test de régression linéaire au risque de $5 \%$.}

\section{RESULTATS}

Les substrats lipidiques (CT, C-HDL, C-LDL, TG) ont été déterminés dans les sera 
de 207 sujets. Le Tableau 2 montre la répartition des valeurs moyennes des substrats lipidiques selon le sexe par la méthode ayant utilisé la formule de Friedewald. Le Tableau 3 fait la répartition des valeurs moyennes des substrats lipidiques selon le sexe par la méthode de précipitation au sulfate de dextrane.

Les valeurs du C-LDL obtenues selon les deux méthodes ont été corrélées. La Figure 1 montre la corrélation entre les valeurs du CLDL selon le calcul par la formule de
Friedewald et la technique de précipitation au sulfate de dextrane chez les 207 sujets. La Figure 2 montre la corrélation entre les valeurs du C-LDL selon le calcul par la formule de Friedewald et la technique de précipitation au sulfate de dextrane chez les hommes. La Figure 3 montre la corrélation entre les valeurs du C-LDL selon le calcul par la formule de Friedewald et la technique de précipitation au sulfate de dextrane chez les femmes.

Tableau 1 : Caractéristiques biométriques de la population d'étude.

\begin{tabular}{lccc}
\hline & $\begin{array}{c}\text { Hommes } \\
(\mathbf{n = 1 1 3})\end{array}$ & $\begin{array}{c}\text { Femmes } \\
(\mathbf{n = 9 4 )}\end{array}$ & $\begin{array}{c}\text { Total } \\
(\mathbf{n = 2 0 7})\end{array}$ \\
\hline $\begin{array}{l}\text { Âge moyen en année } \\
\text { (Moyenne } \pm \text { écart-type) }\end{array}$ & $46,61 \pm 2,97$ & $41,52 \pm 3,18$ & $41,49 \pm 3,08$ \\
$\begin{array}{l}\text { Taille moyenne en mètre } \\
\text { (Moyenne } \pm \text { écart-type) }\end{array}$ & $1,72 \pm 0,06$ & $1,61 \pm 0,07$ & $1,68 \pm 0,07$ \\
$\begin{array}{l}\text { Poids moyen en kilogramme } \\
\text { (Moyenne } \pm \text { écart-type) }\end{array}$ & $73,78 \pm 13,49$ & $71,27 \pm 12,04$ & $72,53 \pm 12,77$ \\
$\begin{array}{l}\text { Indice de masse corporelle moyen } \\
\text { (Moyenne } \pm \text { écart-type) }\end{array}$ & $25,08 \pm 3,96$ & $27,49 \pm 4,39$ & $26,29 \pm 4,18$ \\
\hline
\end{tabular}

Tableau 2 : Valeurs moyennes des substrats lipidiques selon le sexe par la méthode ayant utilisé la formule de Friedewald.

\begin{tabular}{lccc}
\hline & $\begin{array}{c}\text { Hommes } \\
(\mathbf{n}=\mathbf{1 1 3})\end{array}$ & $\begin{array}{c}\text { Femmes } \\
(\mathbf{n = 9 4})\end{array}$ & $\begin{array}{c}\text { Total } \\
(\mathbf{n = 2 0 7})\end{array}$ \\
\hline $\begin{array}{l}\text { Cholestérol total en mmol/l } \\
\text { (Moyenne } \pm \text { écart-type) }\end{array}$ & $4,44 \pm 0,62$ & $4,65 \pm 0,62$ & $4,52 \pm 0,62$ \\
$\begin{array}{l}\text { C-HDL en mmol/l } \\
\text { (Moyenne } \pm \text { écart-type) }\end{array}$ & $1,43 \pm 0,23$ & $1,48 \pm 0,26$ & $1,43 \pm 0,23$ \\
$\begin{array}{l}\text { C-LDL en mmol/l } \\
\text { (Moyenne } \pm \text { écart-type) }\end{array}$ & $2,57 \pm 0,55$ & $2,75 \pm 0,60$ & $2,67 \pm 0,58$ \\
$\begin{array}{l}\text { TG en mmol/l } \\
\text { (Moyenne } \pm \text { écart-type) }\end{array}$ & $0,97 \pm 0,33$ & $0,92 \pm 0,32$ & $0,93 \pm 0,32$ \\
\hline
\end{tabular}


Tableau 3 : Valeurs moyennes des substrats lipidiques selon le sexe par la méthode de précipitation au sulfate de dextrane.

\begin{tabular}{lccc}
\hline & $\begin{array}{c}\text { Hommes } \\
(\mathbf{n = 1 1 3})\end{array}$ & $\begin{array}{c}\text { Femmes } \\
(\mathbf{n = 9 4})\end{array}$ & $\begin{array}{c}\text { Total } \\
(\mathbf{n = 2 0 7})\end{array}$ \\
\hline $\begin{array}{l}\text { Cholestérol total en mmol/l } \\
\text { (Moyenne } \pm \text { écart-type) }\end{array}$ & $4,44 \pm 0,62$ & $4,65 \pm 0,62$ & $4,52 \pm 0,62$ \\
$\begin{array}{l}\text { C-HDL en mmol/l } \\
\text { (Moyenne } \pm \text { écart-type) }\end{array}$ & $1,51 \pm 0,08$ & $1,48 \pm 0,10$ & $1,49 \pm 0,08$ \\
$\begin{array}{l}\text { C-LDL en mmol/l } \\
\text { (Moyenne } \pm \text { écart-type) }\end{array}$ & $2,91 \pm 0,65$ & $2,99 \pm 0,70$ & $2,93 \pm 0,68$ \\
$\begin{array}{l}\text { TG en mmol/L } \\
\text { (Moyenne } \pm \text { écart-type) }\end{array}$ & $0,98 \pm 0,41$ & $0,90 \pm 0,35$ & $0,95 \pm 0,37$ \\
\hline
\end{tabular}

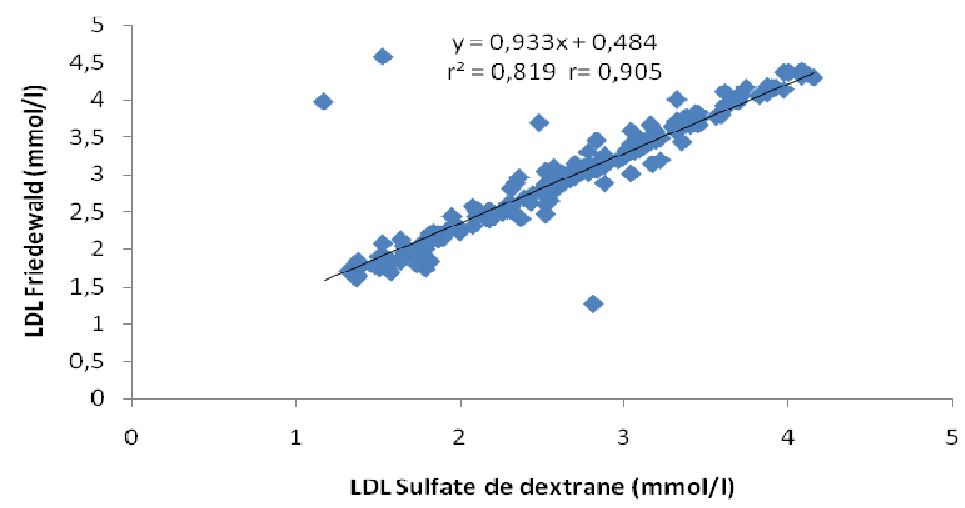

Figure 1 : Corrélation entre les valeurs du C-LDL selon le calcul par la formule de Friedewald et la technique de précipitation au sulfate de dextrane chez les 207 sujets.

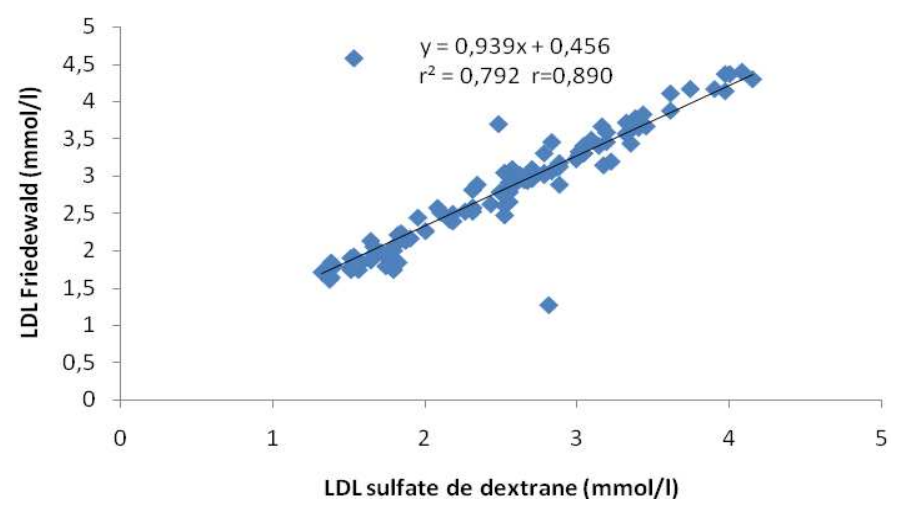

Figure 2 : Corrélation entre les valeurs du C-LDL selon le calcul par la formule de Friedewald et la technique de précipitation au sulfate de dextrane chez les hommes. 


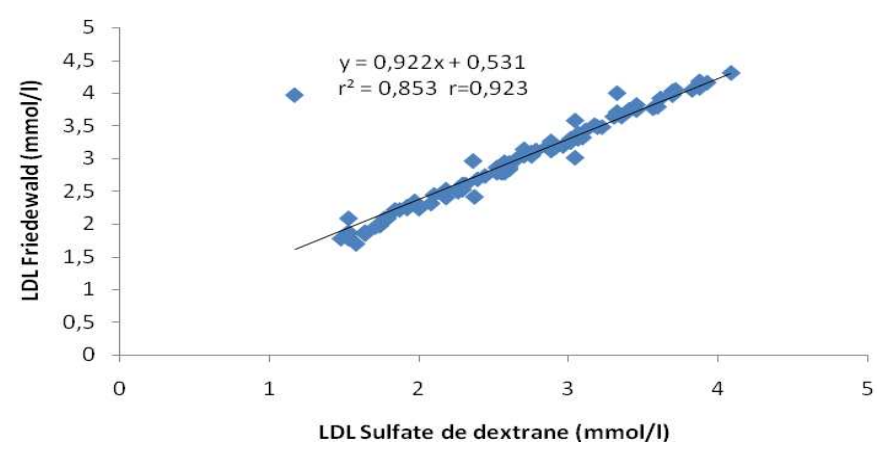

Figure 3 : Corrélation entre les valeurs du C-LDL selon le calcul par la formule de Friedewald et la technique de précipitation au sulfate de dextrane chez les femmes.

\section{DISCUSSION}

Ce travail nous a permis d'une part de déterminer les valeurs du C-LDL par calcul selon la formule de Friedewald et par précipitation au sulfate de dextrane, et d'autre part, de montrer que les deux méthodes avaient une corrélation positive.

De nombreuses techniques de dosage du C-LDL ont été décrites (Rifai et al., 1992) et sont utilisées aussi bien dans les laboratoires de diagnostic que pour la recherche. Mais les méthodes directes de dosage du C-LDL sont les plus couramment utilisées dans les laboratoires des pays développés (Smets et al., 2001 ; Miller et al., 2002 ; McNamara et al., 2006) ; ce qui n'est pas encore le cas dans les pays du tiers monde.

Nous avons obtenu des valeurs moyennes de C-LDL de 2,67 $\pm 0,58 \mathrm{mmol} / 1$ $(2,57 \pm 0,55 \mathrm{mmol} / \mathrm{l}$ pour les hommes et 2,75 $\pm 0,60 \mathrm{mmol} / \mathrm{l}$ pour les femmes) par la formule de Friedewald et de 2,93 $\pm 0,68$ $\mathrm{mmol} / \mathrm{l}(2,91 \pm 0,65 \mathrm{mmol} / \mathrm{l}$ pour les hommes et $2,99 \pm 0,70 \mathrm{mmol} / \mathrm{l}$ pour les femmes) par la méthode de précipitation au sulfate de dextrane. Ces valeurs obtenues par les deux méthodes étaient plus élevées chez les femmes, confirmant ainsi les observations de la littérature (Benlian et al., 2000 ; Bayer et al., 2005). Les valeurs de C-LDL obtenues dans notre étude par les deux techniques étaient inférieures à celles trouvées par Benlian et al. (2000) à l'hôpital St Antoine de Paris sur une population de 442 sujets et qui étaient de 4,21 $\pm 1,38 \mathrm{mmol} / \mathrm{l}$ par ultracentrifugation, de 4,29 $\pm 1,38 \mathrm{mmol} / \mathrm{l}$ par électrophorèse en gel d'agarose et de 3,92 \pm $1,35 \mathrm{mmol} / \mathrm{l}$ par la formule de Friedewald confirmant ainsi les observations selon lesquelles le caucasien a des valeurs de CLDL plus élevées que le mélanoderme (Cappuccio et al., 1997 ; Haffner et al., 1999 ; Sorlie et al.,1999; Cohen et al., 2005). Dans le même ordre d'idées, Mora et al. (2009) aux Etats-Unis d'Amérique avaient trouvé des valeurs moyennes de C-LDL de 3,26 $\pm 0,88$ $\mathrm{mmol} / \mathrm{l}$ par mesure directe et de 3,40 $\pm 0,90$ $\mathrm{mmol} / \mathrm{l}$ par la formule de Friedewald dans une population féminine de 19777 sujets. Si les valeurs de C-LDL trouvées dans notre travail par la formule de Friedewald étaient inférieures à celles obtenues par la technique de précipitation comme l'ont signalé Benlian et al. (2000) par $\beta$-quantification, elles étaient plutôt basses par mesure directe que par la formule de Friedewald selon Mora et al. (2009). L'inexactitude de la formule de Friedewald qui tend à sous-estimer le C-LDL proportionnellement au taux de TG a été donc retrouvée dans notre travail comme l'ont souligné Benlian et Béréziat (2001).

La corrélation entre les deux méthodes de détermination de C-LDL dans notre travail 
était positive avec un coefficient de corrélation $r=+0,905$ pour les 207 sujets $(r=$ $+0,890$ pour les hommes et $r=+0,923$ pour les femmes). Cette corrélation positive et proche de 1 témoigne de l'exactitude de la méthode comme l'ont soulignés Vassault et al. (1999). Benlian et al. (2000), dans une population de 440 sujets, avaient trouvé un coefficient de corrélation de $+0,93$ entre la technique d'électrophorèse en gel d'agarose et l'ultracentrifugation et de $+0,95$ entre la technique d'électrophorèse et le calcul par la formule de Friedewald. De même, Mora et al. (2009) avaient obtenu une corrélation positive $(r=+0,976)$ entre le calcul par la formule de Friedewald et la technique directe de dosage de Roche diagnostics.

Du point de vue de la technique, la précipitation des lipoprotéines au sulfate de dextrane est une $\beta$-quantification non longue et ni laborieuse comme l'électrophorèse en gel d'agarose et l'ultracentrifugation. Dans le procédé qui a été utilisé dans ce travail, il a fallu 50 minutes pour réaliser le dosage du CLDL. Toutefois, cette technique n'est pas dénudée de contraintes. En effet, les réactifs utilisés doivent être préparés au jour le jour et une décantation délicate s'impose pour éviter de contaminer la fraction précipitée avec le surnageant. Le dosage du C-LDL par $\beta$ quantification tout en ne permettant pas d'éliminer les autres formes de cholestérol non C-LDL du milieu réactionnel, limite les erreurs de calcul par la formule de Friedewald qui dépend de trois paramètres (cholestérol total, C-HDL, TG) et qui n'est pas applicable si le taux de TG est supérieur à 4,5 mmol/l. $\mathrm{Au}$ terme de ce travail, la méthode de précipitation au sulfate de dextrane peut donc être utilisée pour le dosage du C-LDL dans le sérum.

\section{REFERENCES}

Bayer P, Veinberg F, Coudrec R, Cherfils C, Cambillau M, Cosson C, Fradin S, Gillery P, Steinmetz J, Legrand A, Egloff M, Beucler I. 2005. Evaluation multicentrique de quatre méthodes de dosage direct du cholestérol LDL. Ann. Biol. Clin., 63: 27-42.
Benlian P, Béréziat G. 2001. Une nouvelle méthode de dosage direct du LDL cholestérol. Revue Française des Laboratoires, 331: 68-69.

Benlian P, Causier C, Hennache G, Khallouf O, Bayer P, Douron F, Carrat F, Coudrec R, Chazouillères O, Bardet J, Bouchard P, Poupon R, Masliah J, Béréziat G. 2000. Comparison of a new method for the direct and simultaneous assessment of LDL- and HDL-cholesterol with ultracentrifugation and established methods. Clin. Chem., 46(4): 493-505.

Cappuccio FP, Cook DG, Atkinson RW, Strazzullo P. 1997. Prevalence, detection, and management of cardiovascular risk factors in different ethnic groups in south London. Heart, 78:555-563.

Cohen J, Pertsemlidis A, Kotowski IK, Graham R, Garcia CK, Hobbs HH. 2005. Low LDL cholesterol in individuals of African descent resulting from frequent nonsense mutations in PCSK9. Nat. Genet., 37: 161-165.

Demacker P, Toenhake-Dijkstra H, de Rijke YB, Stalenhoef AFH, Stuyt PMJ, Willems HL. 1996. On the presumed inaccuracy of the Friedewald formula in hypertriglyceridemic plasma: a role for imprecise analysis. Clin. Chem., 42: 1491-1494.

Friedewald WT, Levy RI, Fredrickson DS. 1972. Estimation of the concentration of low density lipoprotein cholesterol in plasma without use of ultracentrifuge. Clin. Chem., 18: 499-502.

Grundy SM, Bazzarre T, Cleeman J, d'Agostino RB, Sir Hill M, HoustonMiller N, Kannel WB, Krauss R, Krumholz HM, Lauer RM, Ockene IS, Pasternak RC, Pearson T, Ridker PM, Wood D. 2000. Prevention conference V: Beyond secondary prevention; identifying the high-risk patient for primary prevention. Circulation, 101: e3-e11.

Haffner SM, D'Agostino RJr, Goff D, Howard B, Festa A, Saad MF, Mykkanen L. 1999. LDL size in African Americans, Hispanics, and non-Hispanic Whites: the insulin resistance atherosclerosis study. 
Arterioscler. Thromb. Vasc. Biol., 19: 2234-2240.

Iwasaki Y, Matsumaya H, Nakashima N. 2006. Improuved specificity of new homogenous assay for LDL-cholesterol in serum with abnormal lipoproteins. Clin. Chem., 52: 886-888.

Marcovina SM, Albers JJ, Kennedy H, Mei JV, Henderson LO, Hannon WH. 1994. International federation of clinical chemistry standardization project for measurements of apolipoproteins A-I and B. Comparability of apolipoprotein B values by use of International reference material. Clin. Chem., 40: 586-592.

McGowan MW, Artiss JD, Strandbergh DR, Zak B. 1983. A peroxidase-coupled method for the colorimetric determination of serum triglycerides. Clin. Chem., 29(3): 538-542.

McNamara JR, Cohn JS, Wilson PWF, Schaefer EJ. 1990. Calculated values for low-density lipoprotein cholesterol in the assessment of lipid abnormalities and coronary disease risk. Clin. Chem., 36: 36-42.

McNamara JR, Warnick GR, Cooper GR. 2006. A brief history of lipid and lipoprotein measurements and their contribution to clinical chemistry. Clin. Chem. Acta., 369: 158-167.

Miller WG, Waymack PP, Anderson FP, Ethridge SF, Jayne EC. 2002. Performance of four homogeneous direct methods for LDL-cholesterol. Clin. Chem., 48: 489-498.

Mora S, Rifai N, Buring JE, Ridker PM. 2009. Comparison of LDL-cholesterol concentrations by Friedewald calculation and direct measurement in relation to cardiovascular events in 27331 women. Clin. Chem., 55(5): 888-894.

Nauck M, Russel Warnick G, Rifai N. 2002. Methods for measurement of LDLcholesterol: a critical assessment of direct measurement by homogenous assay versus calculation. Clin. Chem., 48: 236254.

Planella T, Cortes M, Martinez-Bru C, Gonzalez-Sastre F, Ordonez-Llianos J. 1997. Calculation of LDL-cholesterol by using apolipoprotein B for classification of nonchylomicronemic dyslipemia. Clin. Chem., 43: 808-815.

Renard C, Ragot C, Tellal S, Vest P, Renaudeau C. 2002. Détermination du cholestérol des lipoprotéines de faible densité: dosage ou calcul. Médecine et Armées, 30: 49-56.

Rifai N, Warnick GR, McNamara JR, Belcher JD, Grinstead GF, Frantz ID. 1992. Measurement of low-density-lipoprotein in serum: a status report. Clin. Chem., 38(1): 150-160.

Smets EM, Pequeriaux NC, Blaton V, Goldschmidt HM. 2001. Analytical performance of a direct assay for LDLcholesterol. Clin. Chem. Lab. Med., 39: 270-280.

Sorlie PD, Sharrett AR, Patsch W, Schreiner PJ, Davis CE, Heiss G, Hutchinson R. 1999. The relationship between lipids/lipoproteins and atherosclerosis in African Americans and Whites: the atherosclerosis risk in communities study. Ann. Epidemiol., 9: 149-158.

Vassault A, Grafmeyer D, De Graeve R, Cohen R, Beaudonnet A, Bienvenu J. 1999. Quality specifications and allowable standards for validation of methods used in clinical biochemistry. Ann. Biol. Clin., 57(6): 685-695.

Wood D, De Backer G, Faergeman O, Graham I, Mancia G, Pyörälä K. 1998. Prevention of coronary heart disease in clinical practice: recommendations of the second joint task force of European and other societies of coronary prevention. Atherosclerosis, 140: 199-270. 